\title{
Structural data bias in the digital age
}

\author{
M.J. Fowler Pells Sullivan Meynink, Australia
}

\begin{abstract}
This paper presents a description of data bias associated with both conventional and digital mapping of rock mass discontinuities. Digital mapping of rock mass discontinuities is now commonplace with, for instance, borehole imaging, LiDAR and photogrammetry becoming standard tools for geotechnical investigations.
\end{abstract}

Rock mass discontinuities are a major control on the design of hard rock slopes and underground openings. Understanding the inherent bias of different sampling methods is an important aspect of building reliable engineering geological models.

\section{Introduction}

The orientation of structural discontinuities in rock is an important input to the engineering assessment of surface and underground structures. All measurements of discontinuities from either boreholes or surface mapping are in effect a sample of a population. Most sampling methods are subject to some form of bias. With the advent of digital mapping techniques such as borehole imaging, laser scanning and photogrammetry some new biases are observed. In addition to bias, some systematic errors are relatively common in data sets.

The recognition of these biases and errors is important in characterising the distribution of discontinuities in the rock mass as the foundation for sound engineering.

\section{$2 \quad$ Orientation bias}

A one-dimensional sample of a rock mass such as a borehole or a mapping scan line is subject to sampling bias (Terzaghi, 1965). The bias is related to the angle between the sampling line and the defect. This concept is demonstrated below in Figure 1 where three discontinuities $A, B$ and $C$ all of equal spacing, with different orientations are intersected by a sample line. It can be observed that Set $A$ is sampled seven times, Set B five and Set C twice. This demonstrates that, although the hypothetical discontinuities are equally distributed, their sampling frequency is partially dependent on the relative angle between the sampling line and the discontinuity.

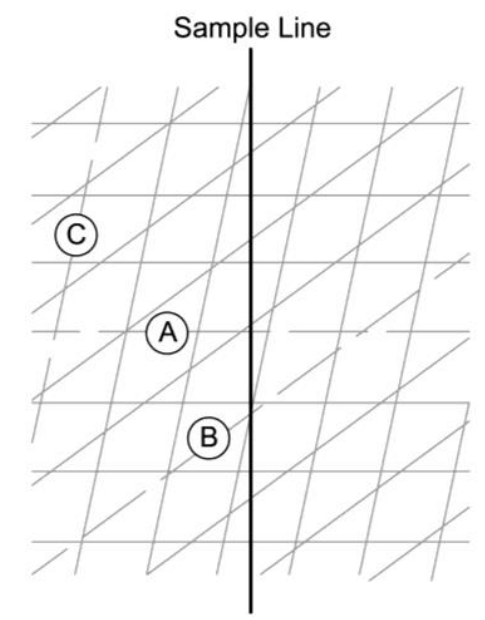

Figure 1 Sampling bias of a one-dimensional sample of a discontinuity is a function of the angle between the line and the defect 
This effect was quantified by Terzaghi (1965) in her method provided for weighting of observations depending on the angle between the discontinuity and the sample line as described below:

$$
N_{90}=N_{\alpha} / \sin \alpha
$$

Where:

$$
\begin{aligned}
& N_{90}=\text { equivalent number of discontinuities if intersected at } 90^{\circ} \text { joint. } \\
& N_{\alpha}=\text { number of intersected discontinuities. } \\
& \alpha \quad=\text { the angle between the sample and the discontinuity. }
\end{aligned}
$$

The effect of this weighting is shown below for a bore hole dipping to the west at $45^{\circ}$.

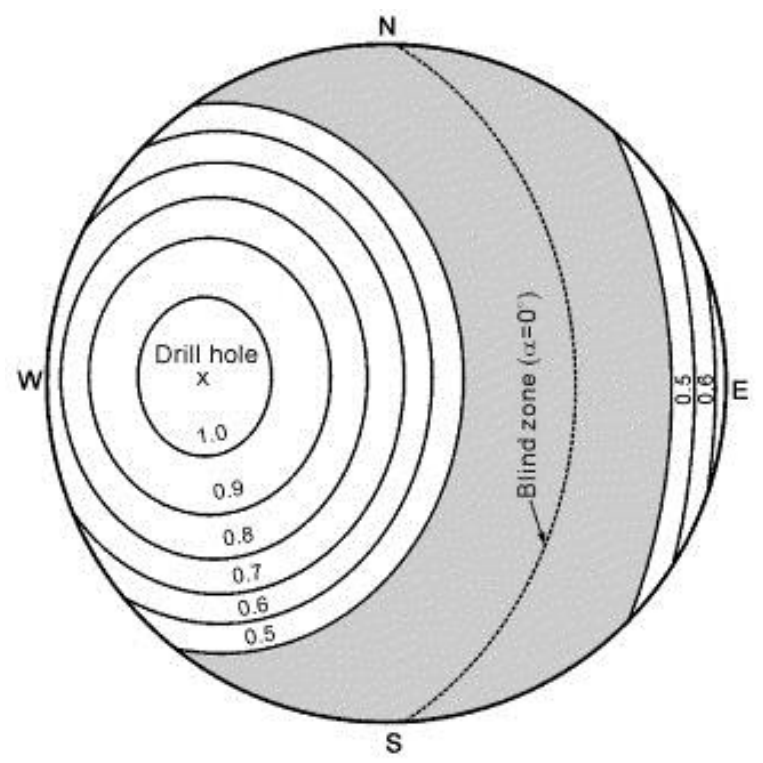

Figure 2 Terzaghi correction factor ' $\alpha$ ' for a drill hole dipping at $45^{\circ}$ to the west

A demonstration of the Terzaghi correction is presented below in Figure 3. Two contoured stereographic projections of the same data are presented, the one on the left is uncorrected and the one on the right has the Terzaghi correction applied. The data is collected from horizontal scan lines. In this instance the blind zone is located in the centre of the stereograph indicated by the red dashed circle. The application of the Terzaghi correction amplifies the set within the blind zone significantly and subtly reduces the concentration of the other observations. The sense of this change is logical. The magnitude is discussed below. 

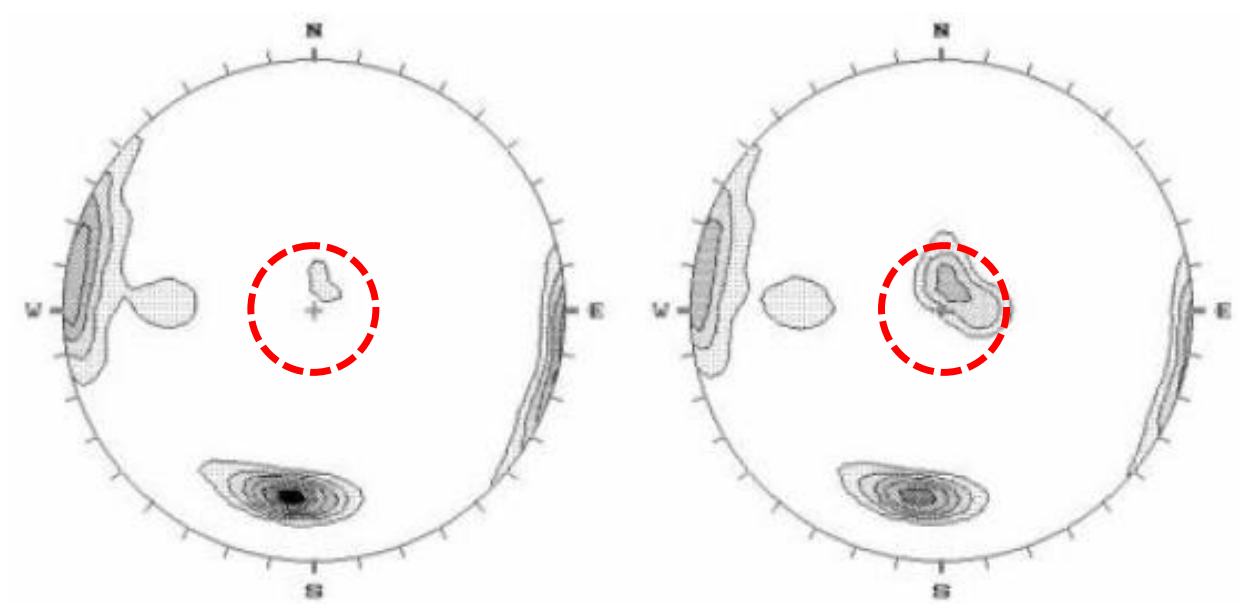

Figure 3 Application of the Terzaghi correction to a mapping horizontal scan lines. The left is uncorrected, right is corrected. The red dashed circle approximates the blind zone (after Rocscience, 2006)

Terzaghi acknowledges that in some cases the remediation offered by the technique in the 'blind zone', that is, near or at $\alpha=$ zero, may not be that useful. This is because in theory the correction tends to infinity as zero is approached and hence may result in unrealistic high weighting. In addition, sampling issues arise, for example; a discontinuity may be missed altogether in the blind zone and therefore cannot be corrected at all or alternatively if a discontinuity is encountered in this zone it may be a random defect as opposed to a member of a persistent set. Either case may lead to a gross misinterpretation of reality.

It is common practice to apply a cut off to the blind zone at say $15^{\circ}$ which limits the weighting. This adds further manipulation to data in the blind zone. Unfortunately all these complications occur precisely where the practitioner is most in the dark.

Probably the most useful observation for the practitioner is not the attempt to quantify the bias but rather its identification. Laing (2005) presents a useful summary of location of notional blind zones associated with various data collection scenarios as presented in Figure 3. 


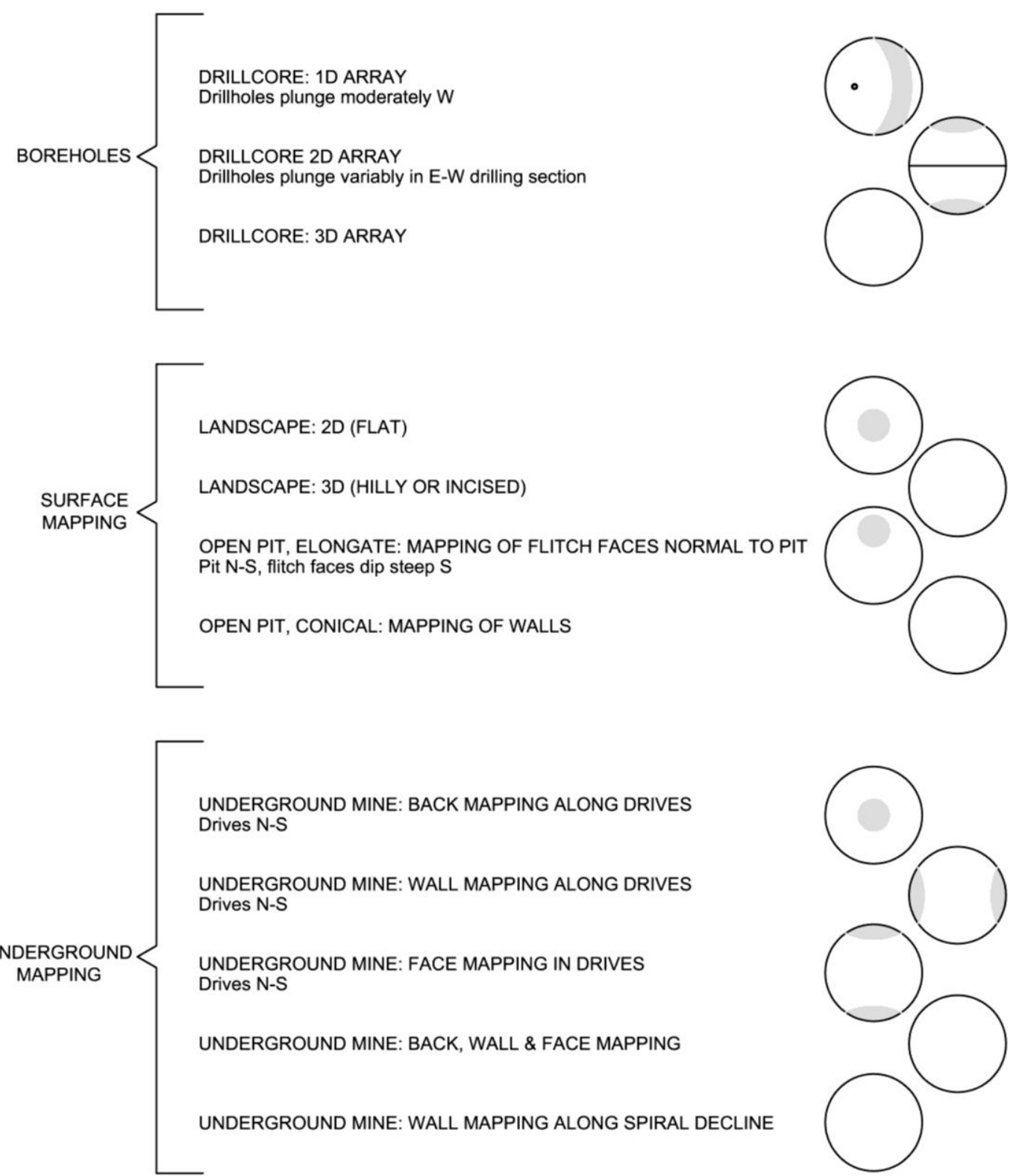

Figure 4 Blind zones for conventional borehole and mapping (after Laing, 2005)

Figure 4 presents three groups of data collection scenario; boreholes, surface mapping and underground mapping. For each scenario, a simplified stereograph is presented where the blind zone is approximated by a light grey shape. Three borehole scenarios are presented. The first is a single borehole drilled to the west with a crescent shape blind zone shown in the east. The second represents two boreholes one drilled to the west and the other to the east, resulting in two small north and south crescent shaped blind zones. The third represents a three-dimensional array of boreholes with no blind zones.

The second group of scenarios relate to surface mapping. The first case represents mapping on a flat surface. Such a surface is blind to horizontal orientations and hence a blind zone is indicated as a central grey circle. When the mapping surface is inclined the grey circle moves. The concept is sufficiently demonstrated and should enable the reader to work through the third group of underground cases.

These simple figures and the learning from this concept provide a very useful basis by which to assess the location of blind zones for any conventional scenario. 


\section{$3 \quad$ Bias and errors associated with core orientation methods}

In addition to the blind zones, errors associated with core orientation are well documented. Sullivan et al. (1992) provides a summary of problems associated with the core orientation including the spear, clay imprint and Craelius methods. In addition to blind zones the following issues are noted:

- Errors in procedures, poor repeatability, isolated orientation runs preventing cross checking.

- Lack of continuity data, resulting in the inability to screen very short discontinuities which would otherwise be ignored from longer important discontinuities.

In the 20 years since this publication, core orientation technology has improved significantly and reduced much of the error. The inability to screen noisy small discontinuities from the larger defects is still present as demonstrated in Figure 5 which presents a stereograph with poles from boreholes plunging to the east. The pattern of defects appears as an amorphous 'shotgun' around the plunge of the drill holes. A clear crescent shaped blind zone is observed in the west. Interestingly there is a subtle borehole-normal blind zone. A similar effect is described in Section 4.
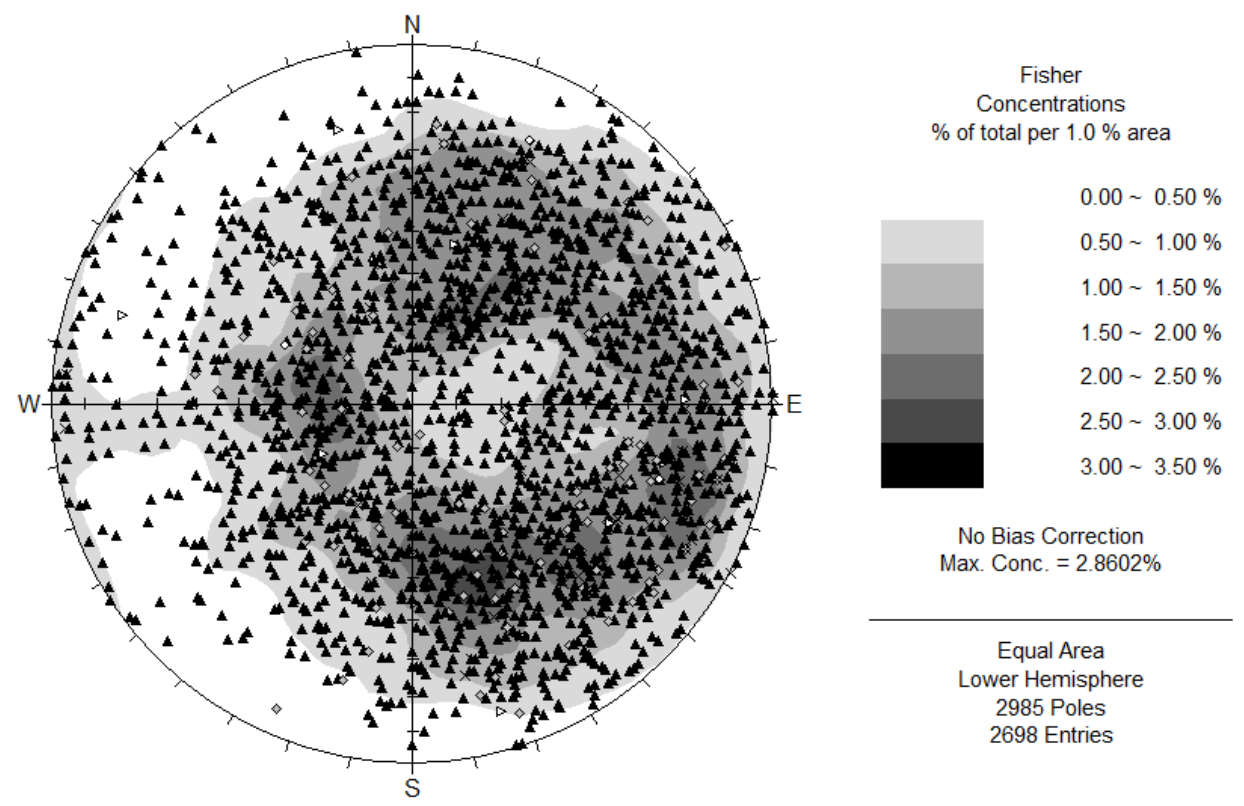

Figure 5 Core orientation data from boreholes drilled to the east from a project in 2013

\section{$4 \quad$ Bias associated with digital methods}

The advent of digital mapping of discontinuities is a step change for rock mechanics with significant advantages. The two primary methods employed in practice are borehole imaging and digital surface mapping via either laser scanning or photogrammetry.

Borehole imaging is a geophysical logging technique involving a downhole image capture of the internal circumference of the borehole wall. This image is mapped for discontinuities by fitting a sine wave to the feature on the unmapped image and then calculations are made to orient in real space. An example of the borehole imaging is presented Figure 6 . The advantages of the technique for rock mechanics purposes are significant and are described elsewhere (Weir, 2012). 


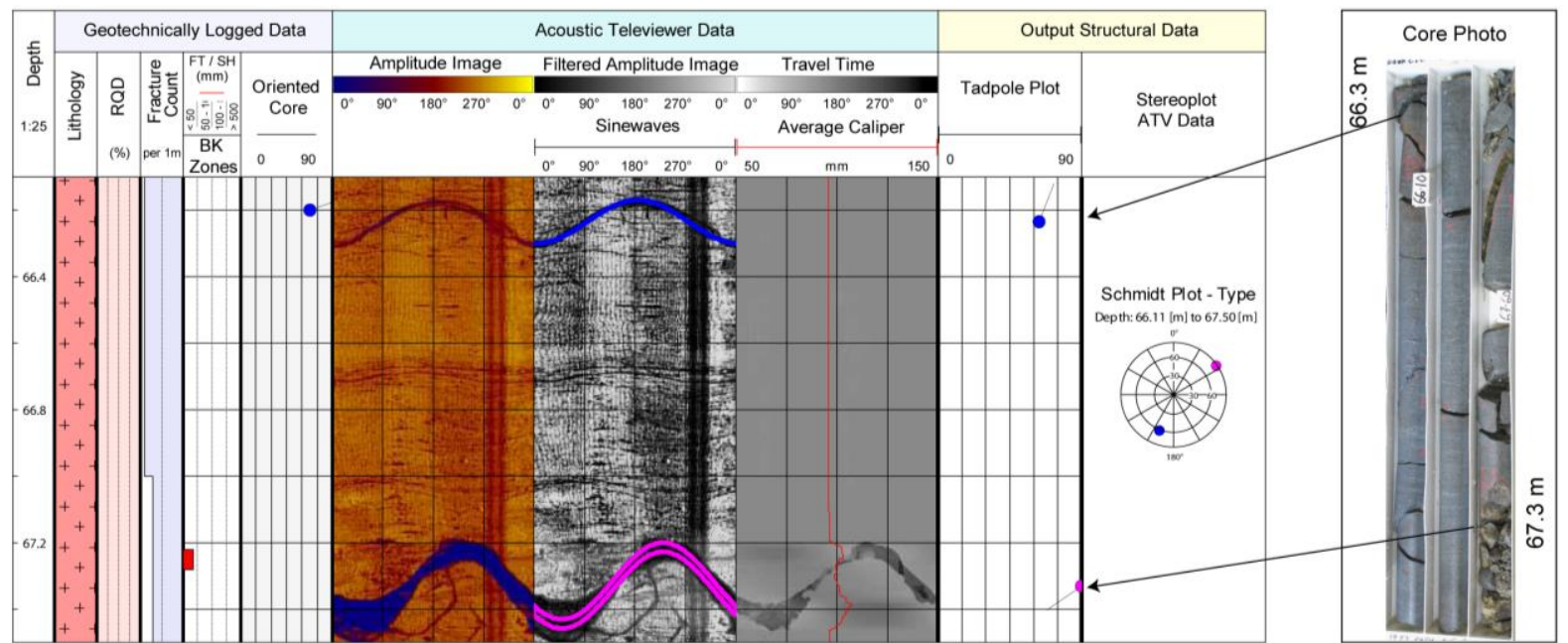

Figure 6 Example of acoustic televiewer borehole image described from left to right, three unwrapped pseudo images of the borehole wall with sine wave in pink and blue (second image), followed by a tadpole plot and stereonet indicating the orientation of the discontinuities and on the right a picture of the core for the same interval (after Weir, 2012)

All sampling methods are subject to some form of bias. In the case of borehole imaging one such bias is the under-representation of discontinuities normal to the axis of the borehole as shown in Figure 7. The conventional blind spot associated with one-dimensional sampling is still present as shown by the grey crescent in Figure 7(a). The mapping of discontinuities involves assigning a sine wave to the unwrapped borehole image. Large sine waves are far more obvious than 'flat' sign waves to the extent that depending on the thickness of the defects and the magnification of the vertical scale used to view the image, 'flat' defects can be under represented, Figure $7(b)$. This results in another blind spot normal to the core axis as indicated by the small grey circle in Figure $7(\mathrm{c})$.

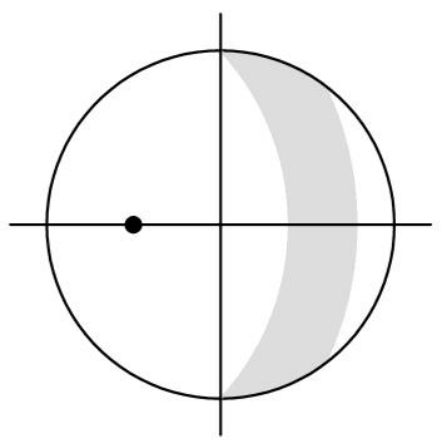

a) Conventional 1D blind spot

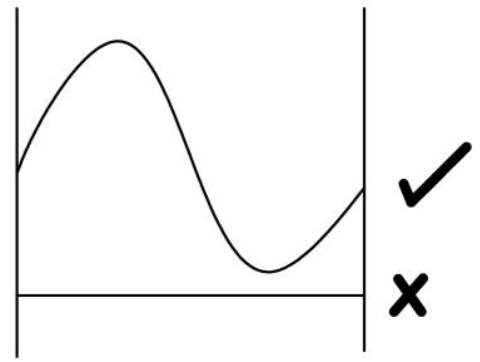

b) Unwrapped borehole image. Flat sine waves less obvious

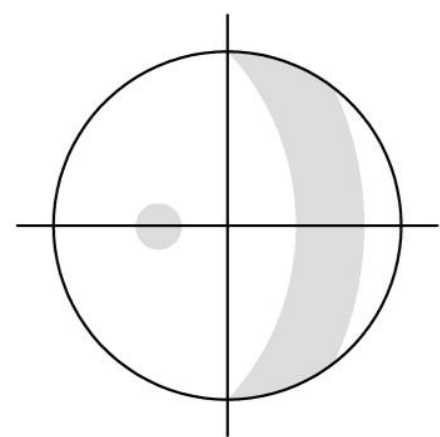

c) Borehole imaging blind spot

Figure 7 (a) Theoretical one-dimension sampling results with a blind spot indicated by the light grey crescent; (b) 'flat' sine waves are more often missed during mapping of the unwrapped borehole images; and (c) this results in an additional blind spot perpendicular to the core axis indicated by the light grey dot

Digital surface mapping comprises the use of technologies such as laser scanning and photogrammetry to build high precision digital terrain models (DTM) of rock exposures. Once a DTM is created, the surface can be mapped by digitising points on the discontinuities and generating a plane of best fit. An example of photogrammetry model is presented in Figure 8 . The presents a photogrammetry model of benched open pit slope with numerous coloured discs representing discontinuities that have been mapped. 


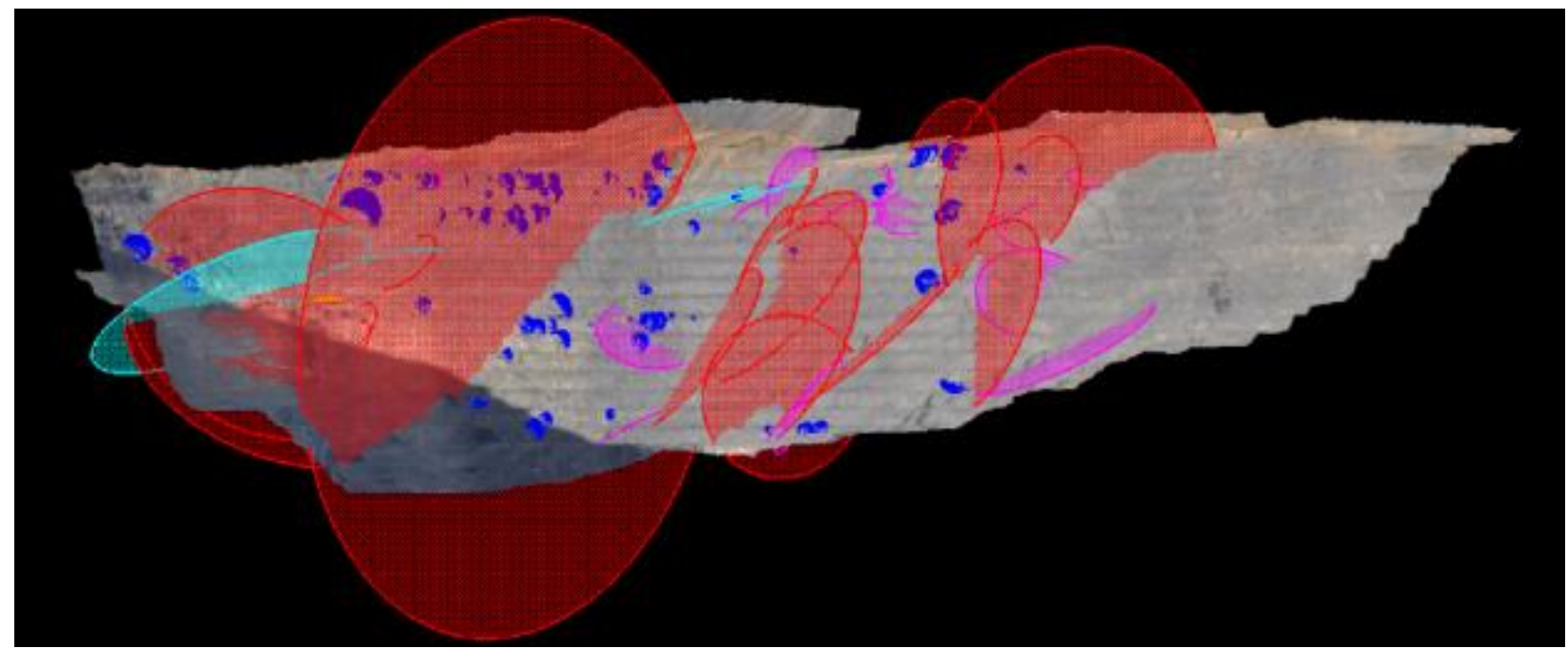

Figure 8 Photogrammetry model of an open pit face. The discs indicate discontinuities that have been mapped to the surface

In the same way that a bias results from the borehole imaging methodology, so does the process of digital mapping introduce bias. There is an inherent preference to map defects that are sub parallel to the face, because these are the facets of the DTM that are best digitised. Discontinuities that are normal to the face are least sampled. Figure 9 demonstrates this concept; 9(a) presents the conventional two-dimensional blind spot; 9 (b) presents the biased mapping of slope parallel defects; 9 (c) represents additional steep east and west located blind spot.

This issue is not universal, it is most pronounced when the rock surfaces are smooth blasted producing planar faces and defects generally shorter than bench height. It is much less a problem when faces are irregular and multifaceted and defects are long.

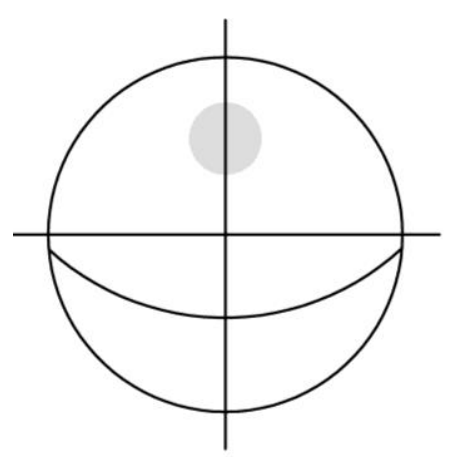

a) Conventional 1D blind spot

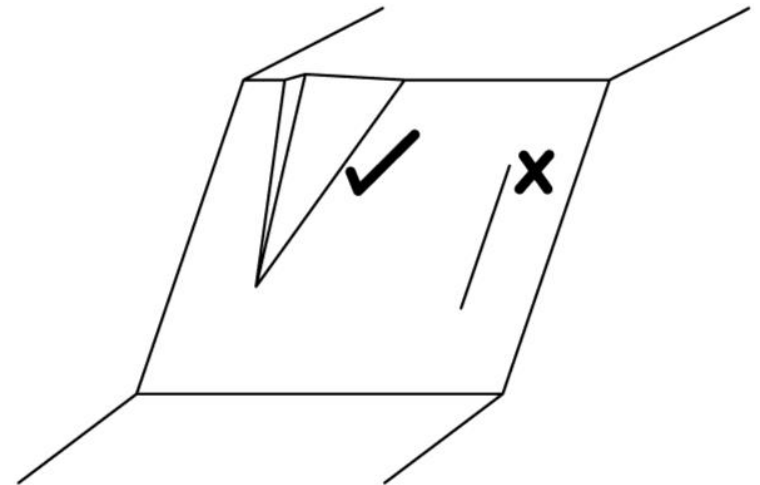

b) Photogrammetry/LiDAR undersamples face normal defects

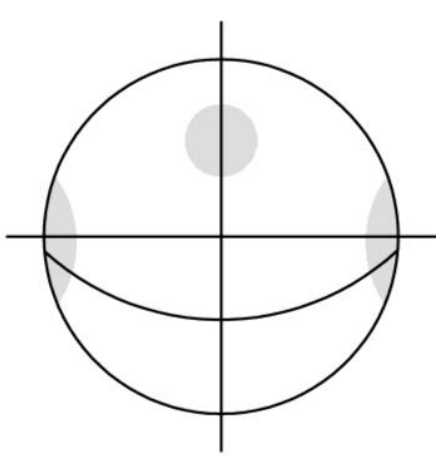

c) Photogrammetry/LiDAR blind spots

Figure 9 (a) Theoretical two-dimension sampling results in a blind spot indicated by the light grey dot; (b) during mapping of DTMs it is more common to be able to obtain orientations from defects sub parallel to the face compared with defects normal to the face; and (c) this results in an additional blind spot normal to the face

This is not an extensive list of biases for digital surface mapping. For example Lato et al. (2010) present bias corrections for the incident angle of a scanning laser. 


\section{Conclusions}

All sampling methods result in some type of bias. Whilst new technologists such as borehole imaging and digital mapping provide significant advantages over analogue technique they too result in their own data bias.

The author recommends the following approach with respect to data bias:

- Review Figure 4 and establish the conventional blind zone.

- Assess the potential for data bias associated with the method and procedure of data acquisition including those associated with new technologies.

- Present stereographic projections indicating the orientation of the borehole or traverses and the location of blind zones.

- Avoid mixing data from different sources.

- Caution should be used when applying corrections including that of Terzaghi, owing to the inherent issues associate with the correction and the fact that is it does not address biases associated with data processing methods.

\section{Acknowledgment}

The author thanks Robert Bertuzzi for reviewing this paper.

\section{References}

Laing, W. (2005) The data Gap in Geology and Its Implication For Resource Estimation, Australian Institute of Geoscientists, Bulletin 42, pp. 37-53.

Lato M.J., Diederichs, M.S. and Hutchinson, D.J. (2010) Bias Correction for View-limited Lidar Scanning of Rock Outcrops for Structural Characterization, Rock Mechanics and Rock Engineering, Springer, Vol. 43, Issue 5, September 2010, pp. 615-628. RocScience (2006) Dips 6.0 Structural data processing software, Toronto, http://www.rocscience.com/products/1/Dips.

Sullivan, T.D., Duran, A. and Eggers, M.J. (1992) The Use and Abuse of Oriented Core in Open Pit Mine Design, Third Large Open Pit Mining Conference, 30 August-3 September 1992, Mackay, Australia, Australasian Institute of Mining and Metallurgy, Parkville, pp. 387-395.

Terzaghi, R.D. (1965) Sources of Error in Joint Surveys, Geotechnique, Vol. 15, pp. 287-304.

Weir, F.M. (2012) The Future of Structural Data from Boreholes, in Proceedings 11th Australia - New Zealand Conference on Geomechanics: Ground Engineering in a Changing World, G. Narsilio, A. Arulrajah, and J. Kodikara (eds), 15-18 July 2012, Melbourne, Australia, Australian Geomechanical Society and New Zealand Geotechnical Society. 
